\title{
The Chemical Mechanism of Pestilences or Coronavirus Disease 2019 (COVID-19)
}

\author{
Dongdong Zhang1, Lin Fang1, Li Wang1, Zhirui Pan'1, Zhongyuan Lai1,2, Mengqu Wu11, Kun Tang1, \\ Ludan Lei ${ }^{1}$, Dahong Qian ${ }^{1,3}$, Zhende Huang ${ }^{1,3}$, Xudong Wang ${ }^{1 *}$, Haibo Chen ${ }^{4}$, Kuo-Chen Chou ${ }^{5 *}$ \\ ${ }^{1}$ Deepblue Academy of Sciences, Deepblue Technology (Shanghai) Co., Ltd., 369 Weining Rd, Shanghai 200050, \\ China; ${ }^{2}$ Department of Physics, Fudan University, Shanghai 200433, China; ${ }^{3}$ School of Biomedical Engineering, \\ Shanghai Jiao Tong University, Shanghai 200240, China; ${ }^{4}$ Deepblue Technology (Shanghai) Co., Ltd., 369 Weining \\ Rd, Shanghai 200050, China; ${ }^{5}$ Gordon Life Science Institute, Boston, MA 02418, USA
}

Correspondence to: Dongdong Zhang, zhangdongdong@deepblueai.com; Lin Fang, fangl@deepblueai.com; Li Wang, wangli194@163.com; Zhirui Pan, panzhr@deepblueai.com; Zhongyuan Lai, abrikosoff@yahoo.com; MengquWu, Mengquwu@gmail.com; Kun Tang, tangk@deepblueai.com; Ludan Lei, 2205498580@qq.com; Dahong Qian, dahong.qian@sjtu.edu.cn; Zhende Huang, Davidhuang10203@sina.com;

Xudong Wang, wangxd@deepblueai.com; Haibo Chen, chenhb@deepblueai.com;

Kuo-Chen Chou; kcchou@gordonlifescience.org or kcchou38@gmail.com

Keywords: Coronavirus Disease, COVID-19, Vaccine, 5-Steps Rule, PseAAC

Received: October 12, $2020 \quad$ Accepted: November 17, $2020 \quad$ Published: November 20, 2020

Copyright $\odot 2020$ by author(s) and Scientific Research Publishing Inc.

This work is licensed under the Creative Commons Attribution International License (CC BY 4.0).

http://creativecommons.org/licenses/by/4.0/

(c) (i) Open Access

\section{ABSTRACT}

In this paper, the chemical mechanism of the coronavirus disease 2019 (COVID-19) has been explored and clearly revealed.

\section{INTRODUCTION}

Around the end of 2019, a new viral species caused large-scale transmissions and infections, discovered in Wuhan (WHO Emergencies Preparedness, Response, 2020) and subsequently around the world (WHO COVID-19 Disease Dashboard, 2020). Symptoms caused include coughing, shortness of breath, and fever. Around $1 \%$ to $5 \%$ (Worldometer, 2020) of confirmed infections have resulted in deaths, mainly due to severe respiratory failure (CDC, 2020). Genealogical tree studies of the new virus strains have later revealed them to be phylogenetically intimate relatives of the Severe Acute Respiratory Syndrome Coronavirus, namely (SARS-CoV), first identified in 2003 [1]. This new virus has been named SARS-CoV-2 by the International Committee on Taxonomy of Viruses (ICTV) (Gorbalenya et al., 2020) on February 11th, 2020 [2-29].

\section{MATERIALS AND METHOD}

For the materials used in this study, see Figure 1 and Figure 2. 
SARS-COV

Bat-CoV

SARS-COV-2

pangolin-CoV

SARS-COV

Bat-CoV

SARS-COV-2

pangolin-CoV

SARS-COV

Bat-CoV

-CoV-2 107

pangolin-CoV

SARS-COV 1119

Bat-CoV 1133

SARS-COV-2 1137

pangolin-CoV 1131

SARS-COV

Bat-CoV

SARS-COV-2

pangolin-CoV

SARS-COV

Bat-Cov

pangolin-CoV

SARS-COV

Bat-CoV

SARS-COV-2

pangolin-CoV

SARS-COV

Bat-CoV

SARS-COV-2

pangolin-CoV

SARS-COV

Bat-CoV

SARS-COV-2

pangolin-Cov

SARS-COV

Bat-CoV

SARS-COV-2

pangolin-CoV

SARS-COV

Bat-CoV

SARS-COV-2

pangolin-CoV

SARS-COV

Bat-CoV

SARS-COV-2

pangolin-CoV

SARS-COV

Bat-CoV

SARS-COV-2

pangolin-CoV

SARS-COV

Bat-CoV

SARS-COV-2

pangolin-CoV

SARS-COV

Bat-CoV

SARS-COV-2

pangolin-CoV

$$
583
$$

643

657

759
QAंLNTLVKQLSSंNF GA ISSVLNंD ILSRLDKVEA AEVQ IDRL I Tं GRLQS LQTYVंTQQL IRAA QA LNT LVKQLSSNE GA I S SVLND I LSR LDKVEAEVO I DRL I T GR LQS LQTY VTOQL IRAA QA LNTLVKOLSSNFGA ISSVLNDILSRLDKVEAEVO IDRLITGRLOSLOTYVTOQLIRAA QALNTLVKOLS SNF GA IS SVLNDILSR LDKVEAEVOIDRLI T GR LOSLOTYVTOQLIRAA

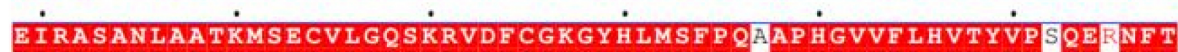

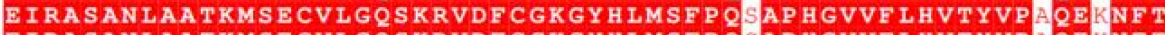

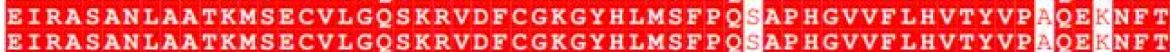

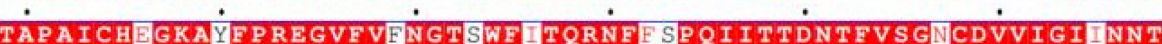
TAPAICHDGKAHFPREGVFVSNGTHWFVTQRNFYEPQI ITTDNTFVSGSCDVVIGIVNNT TAPAI CHDGKAHEPREGVFVSNGTHWFVTORNF YEPQI ITTDNTFVSGNCDVVI G I VNT TAPA I CHEGKAHEPREGVFVSNGTHWFITORNFY EPQI ITTDNTEVSGSCDVVIGIVNNT
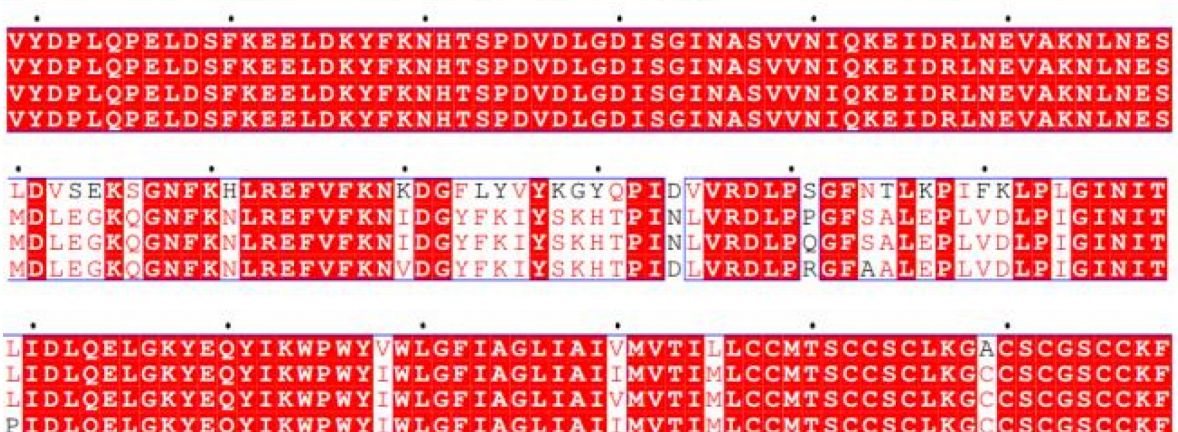

PIDLOEL GKY BOY I KWP WY TWL GF I A I I A I MVTIMLCCMTSCCSCLKGCCSCGSCCKF
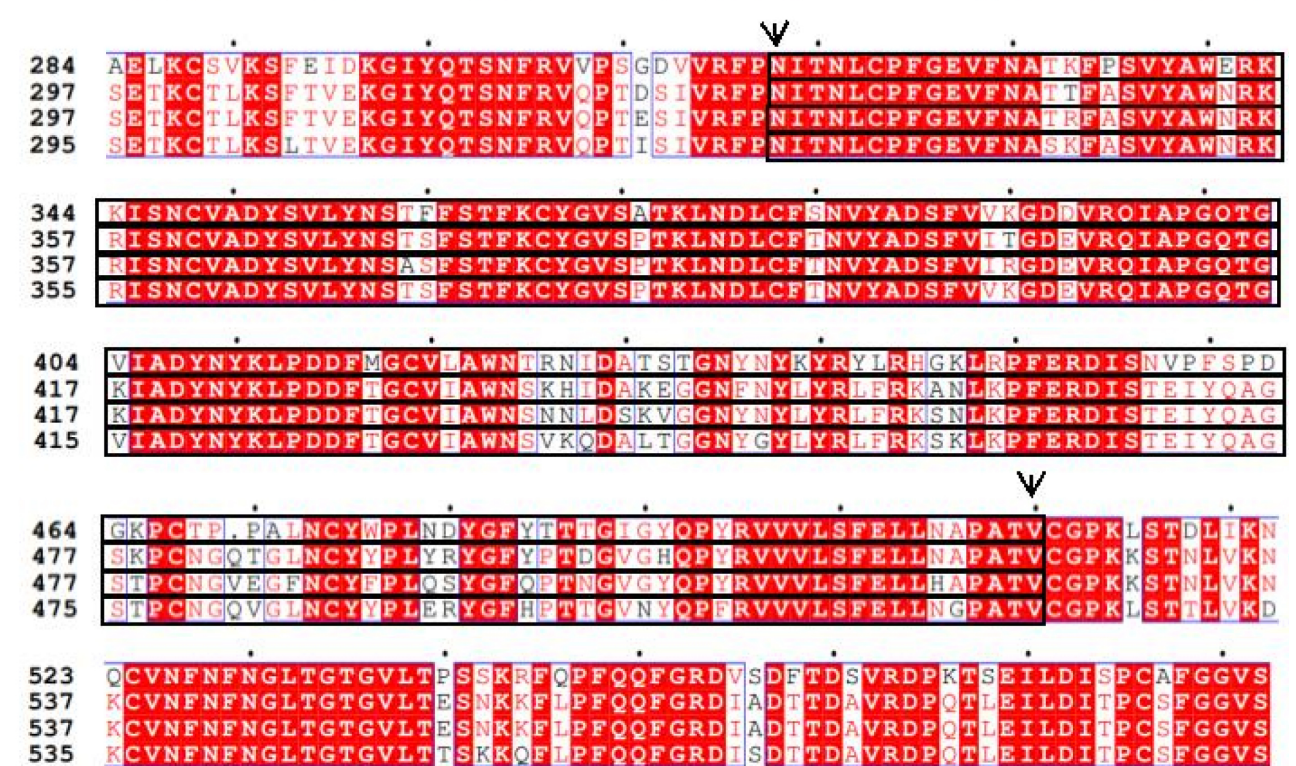

$$
\text { DAंT STTEN }
$$
DSFV

\section{VITP GTNA \\ $V I T P G T N$}

\section{VAVLYQDVNCT \\ VAVLYODVNCT}

IP GTNTSN QVAVLYODVNCTEVPMAI HAEQLTPAWRVYSAGANVEOTRAGCLVGAEHV

657 NNSYBCDIP

655 NNSYBCDIPVGAGICAS
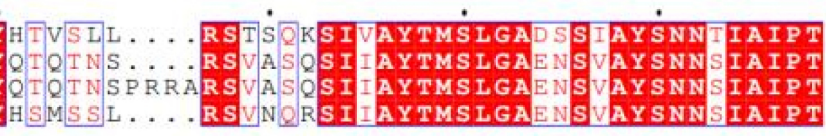

$\begin{array}{ll}699 & \text { NETSIS } \\ 713 & \text { NETIS } \\ 717 & \text { NETIS } \\ 711 & \text { NETIS }\end{array}$

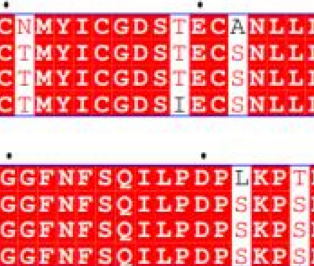

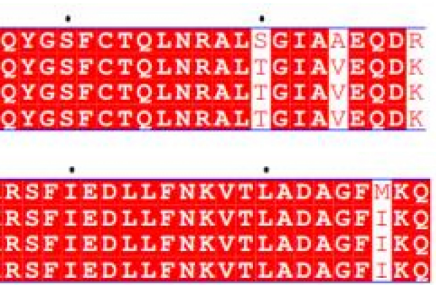




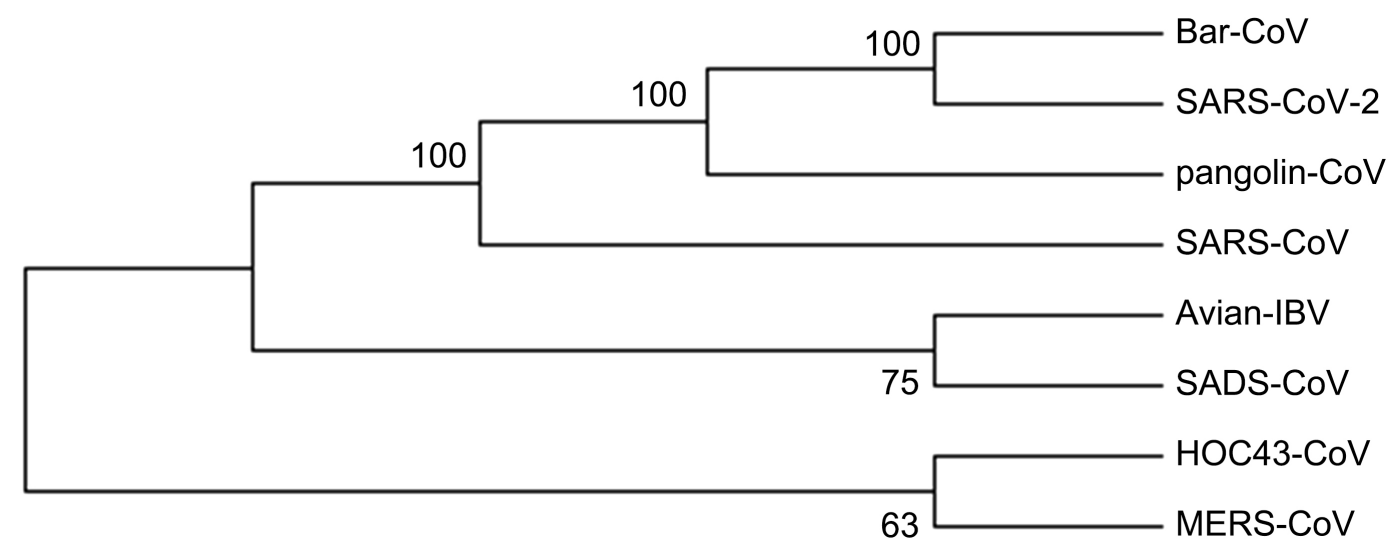

Figure 1. The alignment of the amino acid sequences of the $S$ proteins from 4 coronaviruses and the phylogenetic tree of the genes of the receptor binding proteins from 8 different single strand RNA viruses with the MEGA software. Top: The alignment of the amino acid sequences of the $S$ proteins from 4 coronaviruses, namely Bat Coronavirus RaTG13, Pangolin CoV (GX), SARS-CoV (NC_004718) and SARS-CoV-2 (NC_045512). Receptor Binding Domain (RBD) is labeled with the color boxes and the black arrows indicate the beginning and the end of RBD. 


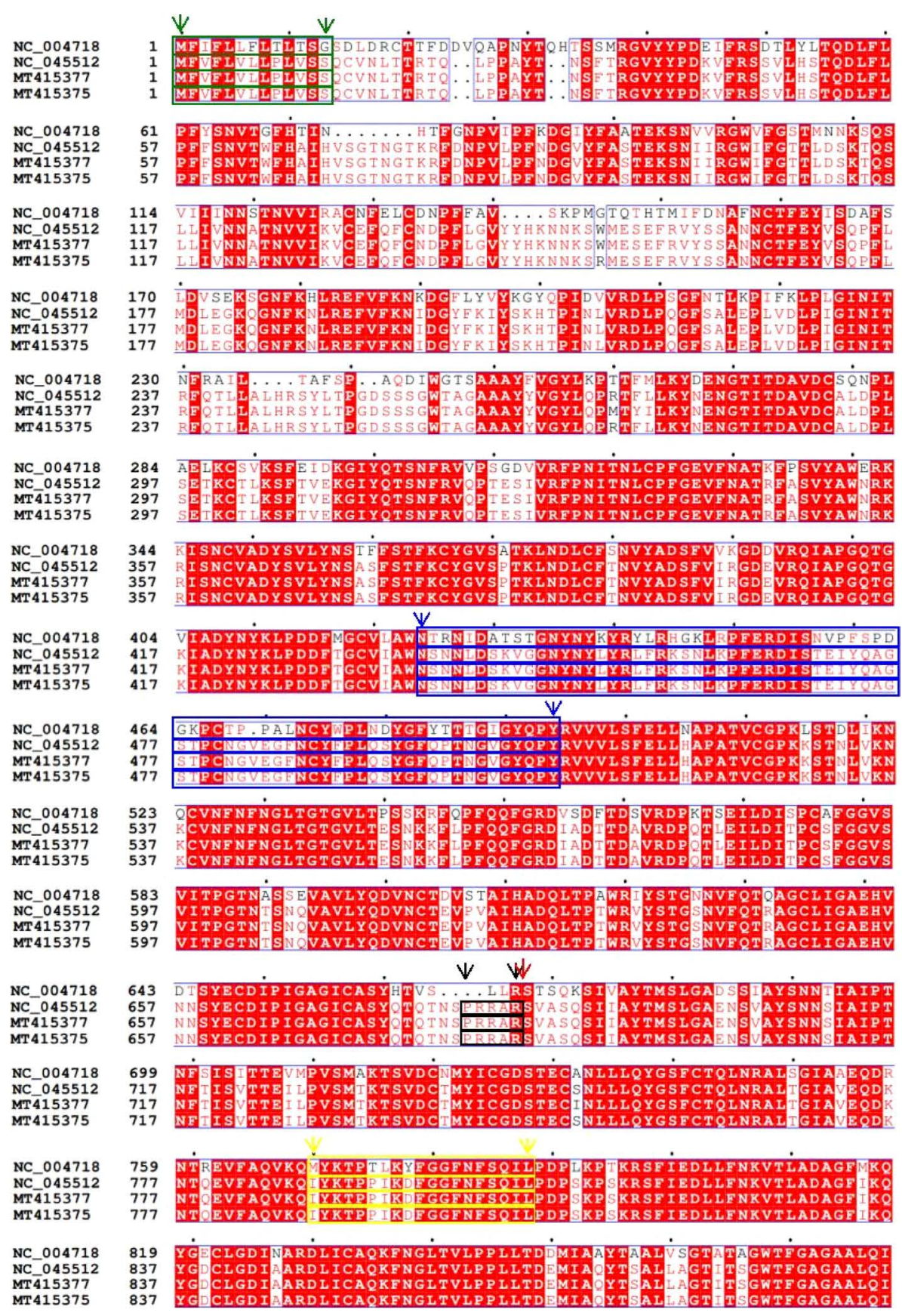

Figure 2. The alignment of the spike (S) protein of 3 strains of SARS-CoV-2 and 1 strain of SARS-CoV (NC_004718.3), SARS-CoV-2 (NC_045512), MT415375 and MT415377. Important functional regions, such as the Signal Peptide (SP), Receptor Binding Motif (RBM), Fusion Peptide (FP) and the inserted amino acid (amino acids PRRA)/host protease recognition site and cleavage site (amino acids RRAR)/cleavage site (between amino acids 685R and 686S) have been marked on the CA pictures. SP: green Box (the green arrows showing the beginning and end of the SP); RBM: blue box (the blue arrows showing the beginning and end of the RBM); PRRAR: black box (the black arrows showing the beginning and end of the inserted/host protease recognition amino acid sites; the red arrow showing the host protease cleavage site between amino acids 685R and 686S) (T. Meng, et al.); FP: yellow box. 
The "Chou's 5-step rules" or "Chou's 5-steps rule" has also been applied because it has been widely and increasingly used by many scientists to study biological sequence analyses [2-56].

To develop a really useful predictor for a biological system, one needs to go through the following five steps: 1) select or construct a valid benchmark dataset to train and test the predictor; 2) represent the samples with an effective formulation that can truly reflect their intrinsic correlation with the target to be predicted; 3) introduce or develop a powerful algorithm to conduct the prediction; 4) properly perform cross-validation tests to objectively evaluate the anticipated prediction accuracy; 5) establish a user-friendly web-server for the predictor that is accessible to the public. Papers presented for developing a new sequence-analyzing method or statistical predictor by observing the guidelines of Chou's 5-step rules have the following notable merits: 1) crystal clear in logic development, 2) completely transparent in operation, 3) easily to repeat the reported results by other investigators, 4) with high potential in stimulating other sequence-analyzing methods, and 5) very convenient to be used by the majority of experimental scientists.

Accordingly, the webserver for the current paper has been currently underway; once done, it will be announced through the addendum of this paper.

\section{CONCLUSION}

The chemical principle proposed in this paper is extremely useful in fighting against the pandemic COVID-19, particularly in developing very powerful vaccine against this kind of horrible disease.

\section{CONFLICTS OF INTEREST}

The authors declare no conflicts of interest regarding the publication of this paper.

\section{REFERENCES}

1. Hyndman, D., Bauman, D.R., Heredia, V.V. and Penning, T.M. (2003) The Aldo-Keto Reductase Superfamily Homepage. Chemico-Biological Interactions, 143-144, 621-631. https://doi.org/10.1016/S0009-2797(02)00193-X

2. Akbar, S., Rahman, A.U., Hayat, M., et al. (2020) cACP: Classifying Anticancer Peptides Using Discriminative Intelligent Model via Chou's 5-Step Rules and General Pseudo Components. Chemometrics and Intelligent Laboratory (CHEMOLAB), 196, Article ID: 103912. https://doi.org/10.1016/j.chemolab.2019.103912

3. Charoenkwan, P., Schaduangrat, N., Nantasenamat, C., Piacham, T. and Shoombuatong, W. (2020) Correction: Shoombuatong, W., et al. iQSP: A Sequence-Based Tool for the Prediction and Analysis of Quorum Sensing Peptides via Chou's 5-Steps Rule and Informative Physicochemical Properties. International Journal of Molecular Sciences, 21, 75. https://doi.org/10.3390/ijms21072629

4. Yang, L., Lv, Y., Wang, S., Zhang, Q., Pan, Y., Su, D., Lu, Q. and Zuo, Y. (2020) Identifying FL11 Subtype by Characterizing Tumor Immune Microenvironment in Prostate Adenocarcinoma via Chou's 5-Steps Rule. Genomics, 112, 1500-1515. https://doi.org/10.1016/j.ygeno.2019.08.021

5. Kabir, M., Ahmad, S., Iqbal, M. and Hayat, M. (2020) iNR-2L: A Two-Level Sequence-Based Predictor Developed via Chou's 5-Steps Rule and General PseAAC for Identifying Nuclear Receptors and Their Families. Genomics, 112, 276-285. https://doi.org/10.1016/j.ygeno.2019.02.006

6. Nosrati, M., Mohabatkar, H. and Behbahani, M. (2020) Introducing of an Integrated Artificial Neural Network and Chou's Pseudo Amino Acid Composition Approach for Computational Epitope-Mapping of Crimean-Congo Haemorrhagic Fever Virus Antigens. International Immunopharmacology, 78, Article ID: 106020. https://doi.org/10.1016/j.intimp.2019.106020

7. Khan, Y.D., Amin, N., Hussain, W., Rasool, N., Khan, S.A. and Chou, K.C. (2020) iProtease-PseAAC(2L): A Two-Layer Predictor for Identifying Proteases and Their Types Using Chou's 5-Step-Rule and General PseAAC. 
Analytical Biochemistry, 588, Article ID: 113477. https://doi.org/10.1016/j.ab.2019.113477

8. Charoenkwan, P., Schaduangrat, N., Nantasenamat, C., Piacham, T. and Shoombuatong, W. (2020) iQSP: A Sequence-Based Tool for the Prediction and Analysis of Quorum Sensing Peptides via Chou's 5-Steps Rule and Informative Physicochemical Properties. International Journal of Molecular Sciences, 21, 75. https://doi.org/10.3390/ijms21010075

9. Roy, T. and Bhattacharjee, P. (2020) A LabVIEW-Based Real-Time Modeling Approach via Chou's 5-Steps Rule for Detection of Abnormalities in Cancer Cells. Gene Reports, Article ID: 100788.

https://doi.org/10.1016/j.genrep.2020.100788

10. Vishnoi, S., Garg, P. and Arora, P. (2020) Physicochemical n-Grams Tool: A Tool for Protein Physicochemical Descriptor Generation via Chou's 5-Step Rule. Chemical Biology \& Drug Design, 95, 79-86.

https://doi.org/10.1111/cbdd.13617

11. Ju, Z. and Wang, S.Y. (2020) Prediction of Lysine Formylation Sites Using the Composition of k-Spaced Amino Acid Pairs via Chou's 5-Steps Rule and General Pseudo Components. Genomics, 112, 859-866. https://doi.org/10.1016/j.ygeno.2019.05.027

12. Khan, S., Khan, M., Iqbal, N., Khan, S.A. and Chou, K.C. (2020) Prediction of piRNAs and Their Function Based on Discriminative Intelligent Model Using Hybrid Features into Chou's PseKNC. Chemometrics and Intelligent Laboratory (CHEMOLAB), 203, Article ID: 104056. https://doi.org/10.1016/j.chemolab.2020.104056

13. Song, C. and Yang, B. (2020) Use Chou's 5-Step Rule to Classify Protein Modification Sites with Neural Network. Scientific Programming, 2020, Article ID: 8894633. https://doi.org/10.1155/2020/8894633

14. Song, C. and Yang, B. (2020) Use Chou's 5-Step Rule to Classify Protein Modification Sites with Neural Network. Scientific Programming, 2020, Article ID: 8894633. https://doi.org/10.1155/2020/8894633

15. Song, C. and Yang, B. (2020) Use Chou's 5-Step Rule to Classify Protein Modification Sites with Neural Network. Scientific Programming, 2020, Article ID: 8894633. https://doi.org/10.1155/2020/8894633

16. Lu, W., Song, Z., Ding, Y., Wu, H., Cao, Y. and Zhang, Y.L. (2020) Use Chou's 5-Step Rule to Predict DNA-Binding Proteins with Evolutionary Information. BioMed Research International, 2020, Article ID: 6984045. https://doi.org/10.1155/2020/6984045

17. Pandey, R.P., Kumar, S., Ahmad, S., Vibhuti, A., Raj, V.S., Verma, A.K., Sharma, P. and Leal, E. (2020) Use Chou's 5-Steps Rule to Evaluate Protective Efficacy Induced by Antigenic Proteins of Mycobacterium Tuberculosis Encapsulated in Chitosan Nanoparticles. Life Sciences, 256, Article ID: 117961. https://doi.org/10.1016/j.lfs.2020.117961

18. Lin, W., Xiao, X., Qiu, W. and Chou, K.C. (2020) Use Chou's 5-Steps Rule to Predict Remote Homology Proteins by Merging Grey Incidence Analysis and Domain Similarity Analysis. Natural Science, 12, 181-198. https://doi.org/10.4236/ns.2020.123016

19. Nguyen, D., Ho-Quang, T., Nguyen Quoc Khanh, L., Dinh-Phan, V. and Ou, Y.Y. (2020) Use Chou's 5-Steps Rule with Different Word Embedding Types to Boost Performance of Electron Transport Protein Prediction Model. IEEE/ ACM Transactions on Computational Biology and Bioinformatics. https://doi.org/10.1109/TCBB.2020.3010975

20. Bouziane, H. and Chouarfia, A. (2020) Use of Chou's 5-Steps Rule to Predict the Subcellular Localization of Gram-Negative and Gram-Positive Bacterial Proteins by Multi-Label Learning Based on Gene Ontology Annotation and Profile Alignment. Journal of Integrative Bioinformatics. https://doi.org/10.1515/jib-2019-0091

21. Chen, Y. and Fan, X. (2020) Use of Chou's 5-Steps Rule to Reveal Active Compound and Mechanism of Shuangshen Pingfei San on Idiopathic Pulmonary Fibrosis. Current Molecular Medicine, 20, 220-230. https://doi.org/10.2174/1566524019666191011160543 
22. Dobosz, R., Mucko, J. and Gawinecki, R. (2020) Using Chou's 5-Step Rule to Evaluate the Stability of Tautomers: Susceptibility of 2-[(Phenylimino)-methyl]-cyclohexane-1,3-diones to Tautomerization Based on the Calculated Gibbs Free Energies. Energies, 13, 183. https://doi.org/10.3390/en13010183

23. Vundavilli, H., Datta, A., Sima, C., Hua, J., Lopes, R. and Bittner, M. (2020) Using Chou's 5-Steps Rule to Model Feedback in Lung Cancer. IEEE Journal of Biomedical and Health Informatics, 21, 1-24. https://doi.org/10.1109/JBHI.2019.2958042

24. Akmal, M.A., Hussain, W., Rasool, N., Khan, Y.D., Khan, S.A. and Chou, K.C. (2020) Using Chou's 5-Steps Rule to Predict O-Linked Serine Glycosylation Sites by Blending Position Relative Features and Statistical Moment. IEEE/ ACM Transactions on Computational Biology and Bioinformatics, 2020, 1-12. https://doi.org/10.1109/TCBB.2020.2968441

25. Mohabatkar, H., Ebrahimi, S. and Moradi, M. (2020) Using Chou's Five-Steps Rule to Classify and Predict Glutathione S-Transferases with Different Machine Learning Algorithms and Pseudo Amino Acid Composition. International Journal of Peptide Research and Therapeutics, 2020, 1-17. https://doi.org/10.1007/s10989-020-10087-7

26. Behbahani, M., Nosrati, M., Moradi, M. and Mohabatkar, H. (2020) Using Chou's General Pseudo Amino Acid Composition to Classify Laccases from Bacterial and Fungal Sources via Chou's Five-Step Rule. Applied Biochemistry and Biotechnology, 190, 1035-1048. https://doi.org/10.1007/s12010-019-03141-8

27. Du, L., Meng, Q., Jiang, H. and Li, Y. (2020) Using Evolutionary Information and Multi-Label Linear Discriminant Analysis to Predict the Subcellular Location of Multi-Site Bacterial Proteins via Chou's 5-Steps Rule. IEEE Access, 8, 56452-56461. https://doi.org/10.1109/ACCESS.2020.2982160

28. Zhao, X.F., Min, Z., Wei, X. and Ju, Y. (2020) Using the Chou's 5-Steps Rule, Transient Overexpression Technique, Subcellular Location, and Bioinformatic Analysis to verify the Function of Vitis vinifera O-Methyltranferase 3 (VvOMT3) Protein. Plant Physiology and Biochemistry, 151, 621-629. https://doi.org/10.1016/j.plaphy.2020.04.015

29. Dutta, A., Dalmia, A., R, A., Singh, K.K. and Anand, A. (2020) Using the Chou's 5-Steps Rule to Predict Splice Junctions with Interpretable Bidirectional Long Short-Term Memory Networks. Computers in Biology and Medicine, 116, Article ID: 103558. https://doi.org/10.1016/j.compbiomed.2019.103558

30. Butt, A.H. and Khan, Y.D. (2018) Prediction of S-Sulfenylation Sites Using Statistical Moments Based Features via Chou's 5-Step Rule. International Journal of Peptide Research and Therapeutics (IJPRT), 17, 711-738.

31. Awais, M., Hussain, W., Khan, Y.D., Rasool, N., Khan, S.A. and Chou, K.C. (2019) iPhosH-PseAAC: Identify Phosphohistidine Sites in Proteins by Blending Statistical Moments and Position Relative Features According to the Chou's 5-Step Rule and General Pseudo Amino Acid Composition. IEEE/ ACM Transactions on Computational Biology and Bioinformatics, 19, 1-21. https://doi.org/10.1109/TCBB.2019.2919025

32. Barukab, O., Khan, Y.D., Khan, S.A. and Chou, K.C. (2019) iSulfoTyr-PseAAC: Identify Tyrosine Sulfation Sites by Incorporating Statistical Moments via Chou's 5-Steps Rule and Pseudo Components. Current Genomics, 20, 306-320. https://doi.org/10.2174/1389202920666190819091609

33. Butt, A.H. and Khan, Y.D. (2019) Prediction of S-Sulfenylation Sites Using Statistical Moments Based Features via Chou's 5-Step Rule. International Journal of Peptide Research and Therapeutics (IJPRT), 19, 71-82.

34. Chen, Y. and Fan, X. (2019) Use Chou's 5-Steps Rule to Reveal Active Compound and Mechanism of Shuangsheng Pingfei San on Idiopathic Pulmonary Fibrosis. Current Molecular Medicine, 19, 511-563.

35. Du, X., Diao, Y., Liu, H. and Li, S. (2019) MsDBP: Exploring DNA-Binding Proteins by Integrating Multi-Scale Sequence Information via Chou's 5-Steps Rule. Journal of Proteome Research, 18, 3119-3132. https://doi.org/10.1021/acs.jproteome.9b00226

36. Dutta, A., Dalmia, A., R, A., Singh, K.K. and Anand, A. (2019) Using the Chou's 5-Steps Rule to Predict Splice 
Junctions with Interpretable Bidirectional Long Short-Term Memory Networks. Computers in Biology and Medicine, 116, Article ID: 103558. https://doi.org/10.1016/j.compbiomed.2019.103558

37. Ehsan, A., Mahmood, M.K., Khan, Y.D., Barukab, O.M., Khan, S.A. and Chou, K.C. (2019) iHyd-PseAAC (EPSV): Identify Hydroxylation Sites in Proteins by Extracting Enhanced Position and Sequence Variant Feature via Chou's 5-Step Rule and General Pseudo Amino Acid Composition. Current Genomics, 20, 124-133. https://doi.org/10.2174/1389202920666190325162307

38. Hussain, W., Khan, S.D., Rasool, N., Khan, S.A. and Chou, K.C. (2019) SPalmitoylC-PseAAC: A Sequence-Based Model Developed via Chou's 5-Steps Rule and General PseAAC for Identifying S-Palmitoylation Sites in Proteins. Analytical Biochemistry, 568, 14-23. https://doi.org/10.1016/j.ab.2018.12.019

39. Hussain, W., Khan, Y.D., Rasool, N., Khan, S.A. and Chou, K.C. (2019) SPrenylC-PseAAC: A Sequence-Based Model Developed via Chou's 5-Steps Rule and General PseAAC for Identifying S-Prenylation Sites in Proteins. Journal of Theoretical Biology, 468, 1-11. https://doi.org/10.1016/j.jtbi.2019.02.007

40. Jun, Z. and Wang, S.Y. (2019) Identify Lysine Neddylation Sites Using Bi-Profile Bayes Feature Extraction via the Chou's 5-Steps Rule and General Pseudo Components. Current Genomics, 20, 592-601. https://doi.org/10.2174/1389202921666191223154629

41. Khan, S., Khan, M., Iqbal, N., Hussain, T., Khan, S.A. and Chou, K.C. (2019) A Two-Level Computation Model Based on Deep Learning Algorithm for Identification of piRNA and Their Functions via Chou's 5-Steps Rule. Human Genetics, 19, 756-799. https://doi.org/10.1007/s10989-019-09887-3

42. Khan, Z.U., Ali, F., Khan, I.A., Hussain, Y. and Pi, D. (2019) iRSpot-SPI: Deep Learning-Based Recombination Spots Prediction by Incorporating Secondary Sequence Information Coupled with Physio-Chemical Properties via Chou's 5-Step Rule and Pseudo Components. Chemometrics and Intelligent Laboratory Systems (CHEMOLAB), 189, 169-180. https://doi.org/10.1016/j.chemolab.2019.05.003

43. Lan, J., Liu, J., Liao, C., Merkler, D.J., Han, Q. and Li, J. (2019) A Study for Therapeutic Treatment against Parkinson's Disease via Chou's 5-Steps Rule. Current Topics in Medicinal Chemistry, 19, 2318-2333. https://doi.org/10.2174/1568026619666191019111528

44. Le, N.Q.K. (2019) iN6-methylat (5-Step): Identifying DNA N(6)-methyladenine Sites in Rice Genome Using Continuous Bag of Nucleobases via Chou's 5-Step Rule. Molecular Genetics and Genomics: MGG, 294, 1173-1182. https://doi.org/10.1007/s00438-019-01570-y

45. Le, N.Q.K., Yapp, E.K.Y., Ho, Q.T., Nagasundaram, N., Ou, Y.Y. and Yeh, H.Y. (2019) iEnhancer-5Step: Identifying Enhancers Using Hidden Information of DNA Sequences via Chou's 5-Step Rule and Word Embedding. Analytical Biochemistry, 571, 53-61. https://doi.org/10.1016/j.ab.2019.02.017

46. Le, N.Q.K., Yapp, E.K.Y., Ou, Y.Y. and Yeh, H.Y. (2019) iMotor-CNN: Identifying Molecular Functions of Cytoskeleton Motor Proteins Using 2D Convolutional Neural Network via Chou's 5-Step Rule. Analytical Biochemistry, 575, 17-26. https://doi.org/10.1016/j.ab.2019.03.017

47. Liang, R., Xie, J., Zhang, C., Zhang, M., Huang, H., Huo, H., Cao, X. and Niu, B. (2019) Identifying Cancer Targets Based on Machine Learning Methods via Chou's 5-Steps Rule and General Pseudo Components. Current Topics in Medical Chemistry, 19, 2301-2317. https://doi.org/10.2174/1568026619666191016155543

48. Liang, Y. and Zhang, S. (2019) Identifying DNase I Hypersensitive Sites Using Multi-Features Fusion and F-Score Features Selection via Chou's 5-Steps Rule. Biophysical Chemistry, 253, Article ID: 106227. https://doi.org/10.1016/j.bpc.2019.106227

49. Liu, Z., Dong, W., Jiang, W. and He, Z. (2019) csDMA: An Improved Bioinformatics Tool for Identifying DNA 6 mA Modifications via Chou's 5-Step Rule. Scientific Reports, 9, Article No. 13109.

https://doi.org/10.1038/s41598-019-49430-4 
50. Malebary, S.J., Rehman, M.S.U. and Khan, Y.D. (2019) iCrotoK-PseAAC: Identify Lysine Crotonylation Sites by Blending Position Relative Statistical Features According to the Chou's 5-Step Rule. PLoS ONE, 14, e0223993. https://doi.org/10.1371/journal.pone.0223993

51. Nazari, I., Tahir, M., Tayari, H. and Chong, K.T. (2019) iN6-Methyl (5-step): Identifying RNA N6-Methyladenosine Sites Using Deep Learning Mode via Chou's 5-Step Rules and Chou's General PseKNC. Chemometrics and Intelligent Laboratory Systems (CHEMOLAB), 19, 1-39. https://doi.org/10.1016/j.chemolab.2019.103811

52. Ning, Q., Ma, Z. and Zhao, X. (2019) dForml(KNN)-PseAAC: Detecting Formylation Sites from Protein Sequences Using K-Nearest Neighbor Algorithm via Chou's 5-Step Rule and Pseudo Components. Journal of Theoretical Biology, 470, 43-49. https://doi.org/10.1016/j.jtbi.2019.03.011

53. Tahir, M., Tayara, H. and Chong, K.T. (2019) iDNA6mA (5-Step Rule): Identification of DNA N6-Methyladenine Sites in the Rice Genome by Intelligent Computational Model via Chou's 5-Step Rule. CHEMOLAB, 189, 96-101. https://doi.org/10.1016/j.chemolab.2019.04.007

54. Wiktorowicz, A., Wit, A., Dziewierz, A., Rzeszutko, L., Dudek, D. and Kleczynski, P. (2019) Calcium Pattern Assessment in Patients with Severe Aortic Stenosis via the Chou's 5-Steps Rule. Current Pharmaceutical Design, 25, 6-31. https://doi.org/10.2174/1381612825666190930101258

55. Yang, L., Lv, Y., Wang, S., Zhang, Q., Pan, Y., Su, D., Lu, Q. and Zuo, Y. (2019) Identifying FL11 Subtype by Characterizing Tumor Immune Microenvironment in Prostate Adenocarcinoma via Chou's 5-Steps Rule. Genomics, 112, 1500-1515. https://doi.org/10.1016/j.ygeno.2019.08.021

56. Zhang, S. and Xue, T. (2020) Use Chou's 5-Steps Rule to Identify DNase I Hypersensitive Sites via Dinucleotide Property Matrix and Extreme Gradient Boosting. Molecular Genetics and Genomics. MGG.

https://doi.org/10.1007/s00438-020-01711-8 